\title{
ON SOME COMPARISON BETWEEN ACCELERATED AGING OF MINERAL OIL UNDER HIGH ELECTRIC FIELD AND HIGH TEMPERATURE
}

\author{
Andrei MANEA ${ }^{1}$, Laurențiu DUMITRAN ${ }^{1}$, Teodora GORJANU ${ }^{1}$, Claudia BORZEA ${ }^{1,2}$ \\ ${ }^{1}$ University Politehnica of Bucharest \\ ${ }^{2}$ Romanian Research and Development Institute for Gas Turbines COMOTI \\ andreimanea.agt@gmail.com, dumitran@elmat.pub.ro, teodora.gorjanu@yahoo.com, \\ claudia.borzea@,comoti.ro,
}

\begin{abstract}
During the operation of electric power transformers, their insulation often made from paper and mineral oil is subjected to thermal, mechanical, and electrical stresses. Therefore, the lifetime of power transformers can be assimilated with their insulation system lifetime. The aim of this paper is to explore and present main degradation aspects for mineral oil, subjected to the action of high electrical field and partial discharge and exposure to thermal stress exclusively, analyzing and pointing out changes in the dielectric properties. Thus, the obtained results indicate that both the thermal and, especially, the electrical stresses produce important variations of the values of the real and imaginary part of the complex relative permittivity, the electrical conductivity, and the loss factor which indicates a severe oil degradation.
\end{abstract}

\section{INTRODUCTION}

Power transformers (PTs) are one of the most important and expensive equipment used in electric energy transmission and distribution grids. Despite the notable progress recorded in the last decades in the designing of such power equipment and operation monitoring devices, their failure is still mainly caused by the breakdown of their insulation [1]. Many previous studies present various aspects related to the mineral oil degradation when subjected to thermal stress, performing accelerated thermal aging at significantly higher temperatures compared to the average operating temperature of a power transformer $[2,3]$. It is generally accepted that thermal stress is the one that causes the most important degradation of the mineral oil-paper insulation system.

Although thermal aging processes of oil-paper insulation are well known, ambiguities still exist regarding the high electrical stress influence on the components of this insulation system. Especially in high voltage power transformers, due to the local intensification of the electric field as a consequence of the presence of certain defects or impurities, partial electric discharges can occur and, under certain conditions, even local oil breakdown, thus leading to very fast decrease of oil dielectric properties.

There is a large number of studies dedicated to detecting and measuring the level of partial discharges in high voltage electrical equipment, however, in very few cases the consequences of this phenomenon on the insulation condition are analyzed. Even in the case of the oil-paper insulation used in power transformers, there are no extensive studies regarding the changes of the dielectric properties of the insulation under the action of the high electric field and of the partial discharges.

Comparing to the accelerated thermal aging method very often used to evaluate the lifetime and remaining lifetime of insulation, there is no recognized experimental method to evaluate the same parameters in the case of high electrical stress. Beyond these aspects, in practice it is accepted that the power transformers' isolation systems (Fig. 1) degrade as a result of the synergistic effect of concomitant action of several aging factors. 


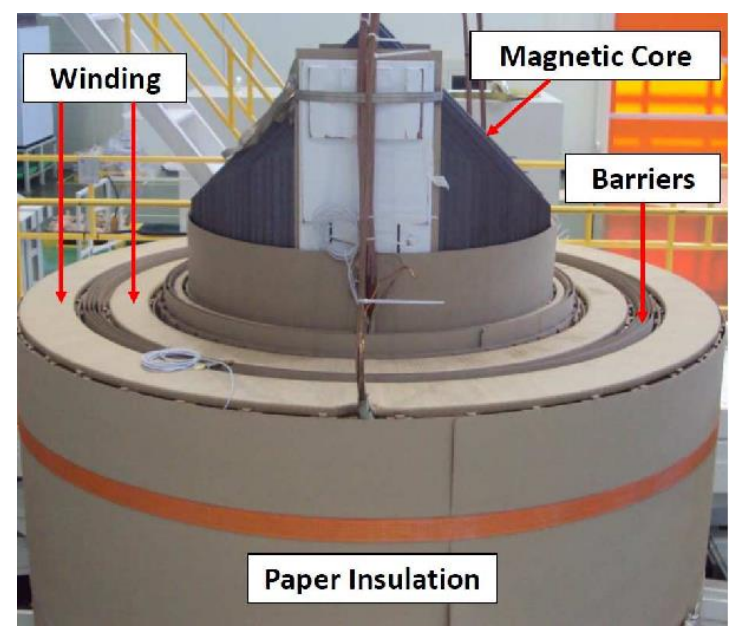

Fig. 1. Power transformer with paper insulation - main elements [4]

The present paper aims to analyze the variation of dielectric properties of mineral oil submitted to accelerated aging under high electric field and partial discharges and make a comparison with the case when the oil is subjected only to thermal stress. Thus, the paper presents a new method which was proposed by the authors for the accelerated aging of electrical insulating liquids under the action of electric field and partial discharges. The experimental results indicate that the dielectric properties of transformer mineral oil experience very important changes that occur in a short time, under the action of high electric field and partial discharges. Compared to the case when the oil is subjected only to thermal stress, these changes in dielectric properties are much more significant.

\section{MINERAL OIL AGING PROCESS}

Regarding the composition of mineral oils, all of them are chemically complex, containing mixtures of several organic compounds. Thus, a variety of chemical reactants and reactions occur because of the combined thermal and electrical actions during PTs operation [5]. It is well known that within PTs, there are several factors that can facilitate and accelerate such chemical reactions. The most important are high temperature and high electric field which at certain locations lead to partial discharge and even local and non-disruptive breakdowns. It is practically impossible to establish an exact mechanism for determining the kinetic parameters of the chemical processes leading to oils' properties degradation.

\subsection{Aging of Transformer Oil under High Electric Field}

Generation of gaseous products in dielectric oils occurs mainly due to the rupture of valence bonds in hydrocarbon molecules. In order to understand how this process occurs, first of all, it is important to identify the energy source that leads to the decomposition of low stability hydrocarbon chains; therefore, the free electrons are the primary source of energy for the breakdown of vulnerable covalent bonds with about $\sim 4 \mathrm{eV}[6]$.

The oils' degradation under electrical and thermal stress takes place in two main stages, namely: 1) Free radical formation - endothermic process with energy/heat absorption and 2) Chemical reactions between the resulting free radicals.

In the kinetics of the first stage, the decisive role is played by the energy transfer due to elastic and non-elastic collisions between free electrons and oil molecules (resulting in excited molecules). Excited molecules produced by the energy absorption in the first stage may undergo a variety of both physical and chemical secondary processes. When these processes start, the electrochemical stability of the oil begins to be affected [6]. 
The mechanism by which high-voltage fields interact with insulating oils [6] shows that the free electrons resulting from ionization process are injected under electric field action at the surface of the metallic conductor. The free electrons in the oil are accelerated by the electric field, resulting in fast-moving electrons. The collision of a fast-moving electron with a hydrocarbon molecule can be either elastic or non-elastic, leading to very distinct results as presented hereinafter. An elastic collision leads to the energy decrease of the free electrons on one hand, and to the increase of the energy of the collided molecule on the other hand (which becomes a high energy molecule). A non-elastic collision produces a free electron with lower energy and an excited (ionized) hydrocarbon molecule. Moreover, vulnerable hydrocarbon molecules decompose and generate pairs of free radicals.

The presence of free radicals leads to the sensitive alteration of dielectric properties of these liquids. In the process of energy transfer activation, a special role is played by the oxygen dissolved in oil. This one has the special ability to capture electrons, forming ionized species of oxygen $\left(\mathrm{O}_{2}\right)$ with long lifespan [7]. The latter returns to its original state by means of light emissions in the ultraviolet spectrum (corona discharges), and consequently favoring the generation of free radicals. It was found that the oxygen dissolved in oil, as well as the copper conductor coming into contact with oil, are accelerating factors of oil properties degradation [5].

The free radicals formed in the first stage usually have a short lifespan and recombine (initiating secondary stage) form various other products, which are not found in the initial oil chemical composition. Some of these products are gaseous, such as hydrogen $\left(\mathrm{H}_{2}\right)$, methane gas $\left(\mathrm{CH}_{4}\right)$, ethylene $\left(\mathrm{C}_{2} \mathrm{H}_{4}\right)$ and ethane $\left(\mathrm{C}_{2} \mathrm{H}_{6}\right)[5,6]$. All these flammable gases can be formed in the second stage and are part of the gaseous compounds causing degradation of the insulation system. This can jeopardize the safe operation of power transformers.

In addition, it has been found that the gas bubbles formed in oil favor the generation of partial discharges which have a significant impact on the dielectric properties of mineral oil. Also, since these gas bubbles accumulated in the liquid are elongated along the electric field lines, another process of decomposition of dielectric liquids is favored [5,8]. Other studies indicate that bubbles' main effect is increasing electrons emission from metals due to electrical field concentration and the bubbles which are produced on the surface of the metallic electrodes [8]. Also, the microscopic metallic points which are located on the electrode surface act as electric field concentrators and give rise to corona discharge which can vaporize the oil and contribute to the dissociation of hydrocarbon molecules and other chemical products $[6,7]$.

\subsection{Aging of Transformer Oil under High Temperature Exclusively}

Beside the electrical field, high temperature (heat produced by the magnetic core and windings) is an important factor that contributes to oil degradation. Under normal operating conditions of the PTs, the rotational and vibrational energies of the hydrocarbon chains of oil are excited, and the breaking of a covalent bond occurs only when a sufficiently high energy level is reached. However, weakly bound molecules can occasionally accumulate enough energy to reach a high enough energy level to initially decompose into two free radicals that can further react, as described above. When subjected to a high temperature for a long time, the hydrocarbons chains of the oil break down into a series of carbon and oxygen compounds. Chemical reactions that occur can be either cracking or breaking. In addition, the high temperature accelerates the oxidation process, increases the mobility of free radicals generated by the gases formed in the oil and thus favors the secondary chemicals' random reactions, preceding the formation of decomposition products [9].

Oxygen influences the aging processes of mineral oils, forming direct chemical bonds (oxidation) with their molecules. The oxidation reaction of hydrocarbons begins with the formation of hydroperoxides, which in one direction result in oxides, and in the other, ketones 
which result in acids and further other soluble or insoluble substances, all with negative effect on the properties of the oils.

The rate of oxidation, the intensity of the phenomenon and the products that are formed depend on the chemical composition of the oil, the oxidation conditions (temperature, pressure, air contact surface) and the presence of oxidation promoting or inhibiting substances. Thus, the phenomenon of oil oxidation is the main source that favors the appearance of acids which, on the one side, are dissociated in the presence of water - favoring the increase of $\mathrm{H}^{+}$ion concentration and, on the other side, increase the oil absorption capacity of water.

Therefore, the solubility of water in oil is higher in the case of degraded oils [10]. When the concentration of dissolved oxygen in the oil is as high as in an open transformer, the accelerated oxidation process by temperature, humidity or chemicals forms peroxides that dissociate into free radicals. Furthermore, they behave as initiators of chain oxidation reactions, which lead to the worsening of the properties of electrical insulating materials [9].

The degradation process is also influenced by oil coming into contact with metals with catalytic effect. The main reactions that lead to the degradation of mineral oils are the breaking of the $\mathrm{C}-\mathrm{C}$ bonds with the formation of an alkane and a smaller alkene (cracking reaction itself), resulting in new hydrocarbons $\left(\mathrm{CH}_{4}, \mathrm{CH}_{2}, \mathrm{C}_{2} \mathrm{H}_{6}, \mathrm{C}_{2} \mathrm{H}_{4}\right)$, as well as other by-products such as $\mathrm{CO}, \mathrm{H}_{2}$ and $\mathrm{H}_{2} \mathrm{O}$ [10], all of them jeopardizing the safe operation of power transformers.

\section{EXPERIMENTAL SETUP, SAMPLES AND METHOD}

\subsection{Accelerating Thermal Aging Method and Experimental Setup}

The experiments were carried out on MOL mineral oil, starting with preparing samples (15 in total - 5 for each aging temperature). The samples (Fig. 2a) consist of stainless-steel aging cells filled with $240 \mathrm{ml}$ of oil. During the aging process, all the cells were closed and insulated with high temperature resistant silicone sealant and then placed in a laboratory oven. The study aims to accelerate aging of mineral oil at three different temperatures, namely $135^{\circ} \mathrm{C}(1000 \mathrm{~h})$, $155^{\circ} \mathrm{C}(1000 \mathrm{~h})$ and $175^{\circ} \mathrm{C}(1000 \mathrm{~h})$. For this, stainless steel aging cells like the one in Fig. $2 \mathrm{~b}$ were used [11].
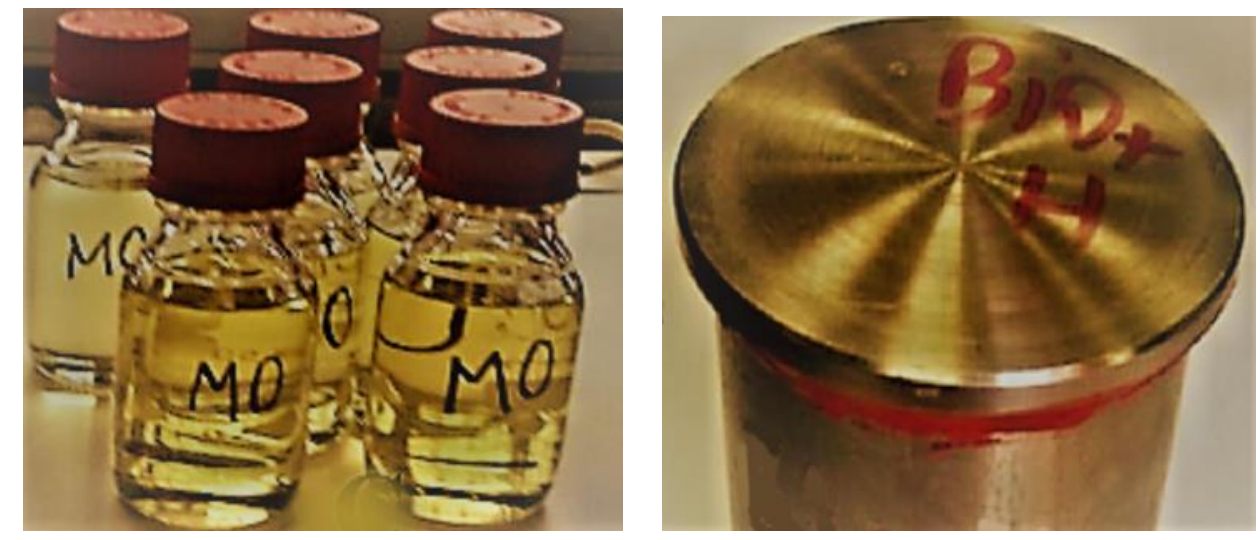

Fig. 2. a) Samples (Mol mineral oil); b) Thermal accelerated ageing cell

To evaluate the degradation due to the acceleration of the thermal process at every 200 hours a cell was extracted and analyzed looking to determinate the values of the following diagnostic factors: the complex relative permittivity $\left(\varepsilon_{\mathrm{r}}{ }^{\prime}\right.$ and $\varepsilon_{\mathrm{r}}$ "'), the loss factor $(\operatorname{tg} \delta)$ and the conductivity $(\sigma)$; Novocontrol dielectric spectrometer, equipped with measuring cells for liquid samples of about $1.8 \mathrm{ml}$ were used for the experimental determination [11].

The samples were subjected to an alternating voltage with an effective value of $1 \mathrm{~V}$ and frequencies in the range $2 \cdot 10^{-3} \mathrm{~Hz}$ to $10^{5} \mathrm{~Hz}$. 


\subsection{Electric Field Accelerating Aging Method and Experimental Setup}

In accordance with the phenomenon presented, a special aging cell was designed so that the applied electric field to facilitate gas bubble formation, and to subsequently lead to partial discharges. The aging cell was designed in such a way as to avoid total oil electric breakdown between the electrodes.

The accelerated aging method is based on the effect of high strength electrical field applied to a small volume ( $\sim 8 \mathrm{ml})$ of mineral oil. The aging cell () is realized of a special glass test tube with the inner diameter of $14 \mathrm{~mm}$ and $130 \mathrm{~mm}$ in height. The thickness of the glass wall is $1.25 \mathrm{~mm}$.

The experimental setup presented in Fig. 4 can supply up to five aging cells at the same time. Electric voltage applied between the central metallic electrode and the sodium chloride solution can be adjusted from 0 to $50 \mathrm{kV}$; during voltage application the aging cells ware careful monitored also including a video camera to record.

The aging cell in Fig. 3 a consists of: 1 - fine tube for gases discharge, 2 - upper sealing plug, 3 - test tube, 4 - inner centering plug, 5 - brass inner electrode, 6 - mineral oil. The experimental setup is presented in Fig. $3 \mathrm{~b}$ and consists of: 1 - adjustable transformer for setting the voltage value; 2 - voltmeter; 3 - high voltage transformer; 4 - aging cells.
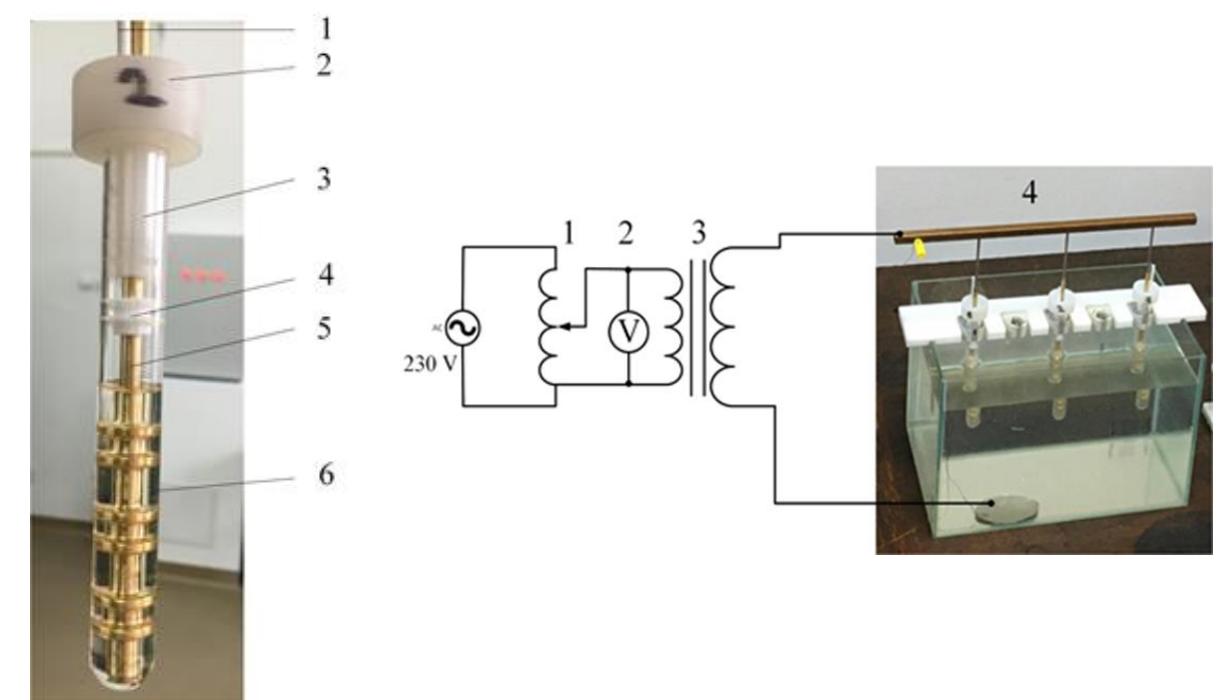

Fig. 3. a) Aging cell: 1 - fine tube for gases discharge, 2 - upper sealing plug, 3 - test tube, 4 - inner centering plug, 5 - brass inner electrode, 6 - mineral oil; b) Experimental setup: 1 -adjustable transformer for setting the voltage value; 2 - voltmeter; 3 - high voltage transformer; 4 - aging cells

Similarly to thermal aging case, the tests were conducted on samples of MOL mineral oil used for power transformers insulation systems. Before being inserted into the aging cell, the oil was conditioned for 72 hours in a forced flow oven at $60^{\circ} \mathrm{C}$.

For assessing the accelerated aging process of mineral oil under the influence of high strength electric fields and partial discharges, the dielectric properties of the oil samples were measured using dielectric spectroscopy. For this purpose, we used the same Novocontrol spectrometer with the special measuring cell for dielectric liquids, with a volume of $\sim 1.8 \mathrm{ml}$. The real and imaginary parts of the complex relative permittivity $\left(\varepsilon_{\mathrm{r}}{ }^{\prime}\right.$ and $\varepsilon_{\mathrm{r}}{ }^{\prime}$ '), dielectric loss factor $\operatorname{tg} \delta$ and the conductivity $(\sigma)$ were measured in a frequency range of $2 \cdot 10^{-3}$ to $10^{5} \mathrm{~Hz}$.

Three oil samples were taken out from each aging cell, kept in an oven at $50{ }^{\circ} \mathrm{C}$ for 12 hours and then measured. Each experimental value was obtained as the average of the three measured results.

\section{EXPERIMENTAL RESULTS AND DISCUSSION}


The variation of real and imaginary parts of complex relative permittivity, loss factor and conductivity as a function of aging time, $\tau$, for $f=2 \cdot 10^{-3} \mathrm{~Hz}$ are displayed for MOL mineral oil accelerated aged under electric field action and thermal stress, respectively.

Knowing that the values of the quantities $\varepsilon_{r}^{\prime}, \varepsilon_{r}^{\prime \prime}, \operatorname{tg} \delta$ and $\sigma$ at low frequencies values (less than $10^{-1} \mathrm{~Hz}$ ) best characterize the aging state of transformer oils [3, 12], our attention is focused to the examination of the curves corresponding to lower values of the electric field frequency $\left(2 \cdot 10^{-3} \mathrm{~Hz}\right)$.

Fig. $4 \mathrm{a}$ and Fig. $4 \mathrm{~b}$ show the variation of conductivity, $\sigma$, as a function of aging time, $\tau$, for $f=2 \mathrm{mHz}$, at a measurement temperature $T=25^{\circ} \mathrm{C}$. It can be observed that the electric conductivity has an important increase with aging time, $\tau$, when the oil is subjected to the high strength electric field aging process, comparing to the thermal aging process exclusively. These values indicate that in the case of accelerated ageing under high electric field, it results a very high concentration of free charge carriers in the oil and therefore an important increase of conductivity, 100 times higher than the case of thermal ageing. This indicates a significant deterioration of the dielectric properties of the oil, which shows that the action of high-power electric fields and partial discharges can degrade the dielectric properties of the mineral oil extremely fast.
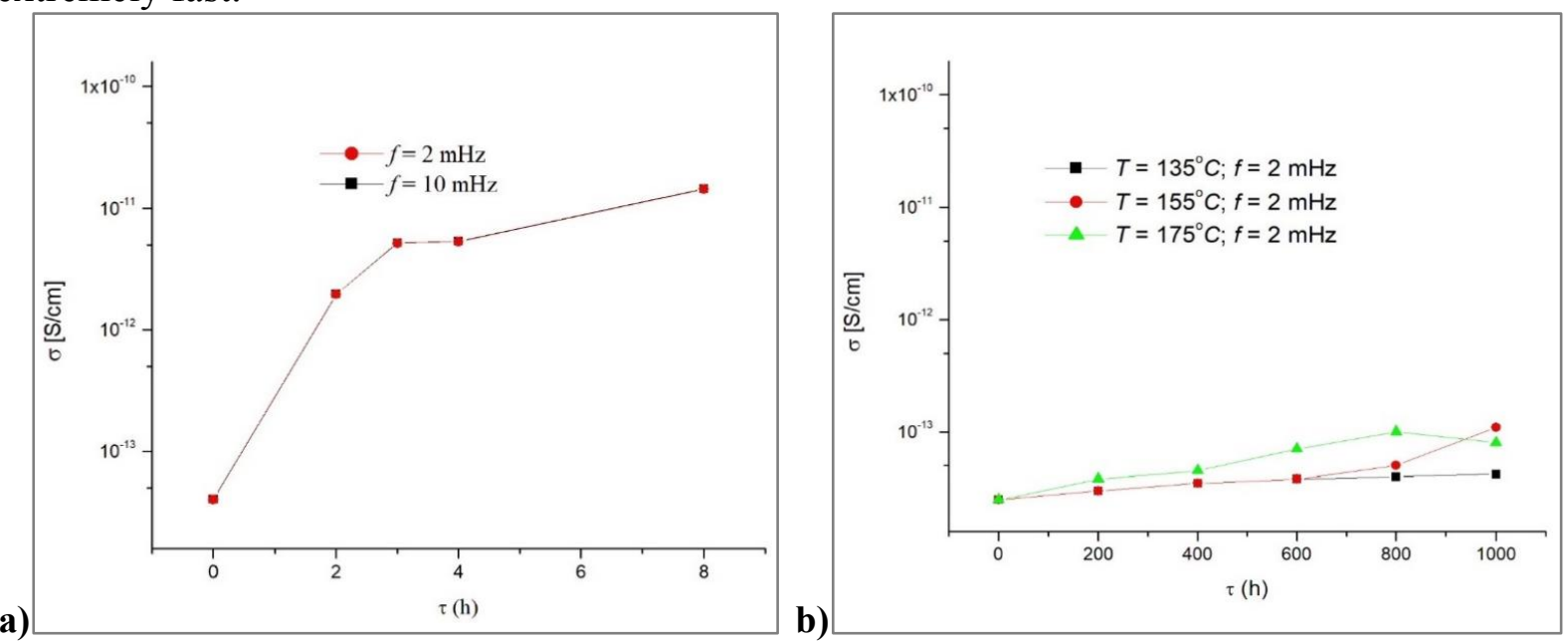

Fig. 4. Variation of conductivity function of aging time for a) Aging applied voltage $U=13 \mathrm{kV}$; b) Thermal aging temperatures $T=(135,155,175)^{\circ} \mathrm{C}$

Fig. 5a and Fig. 5b show the variation of dielectric loss factor, $\operatorname{tg} \delta$, as function of aging time, $\tau$, for $f=2 \mathrm{mHz}$, at a measurement temperature $T=25^{\circ} \mathrm{C}$. Additionally, the variation of the loss factor with aging time requires a special observation. It can be observed that in the case of thermally and electrically accelerated aging, the values of the loss factor increase, but a different behavior is noticed in the case of accelerated aging under electric field, where a slight decrease after 2 hours can be noticed. This is probably due to the different stages of the degradation processes, namely in the case of accelerated thermal aging the degradation process is slower and does not come to capture the subsequent phenomena found in the case of accelerated aging under electric field, where due to high concentration of polar free radicals, it results in a very high increase in $\varepsilon_{\mathrm{r}}^{\prime}$ value and consequently a decrease in the calculated value of $\operatorname{tg} \delta$. 
a)

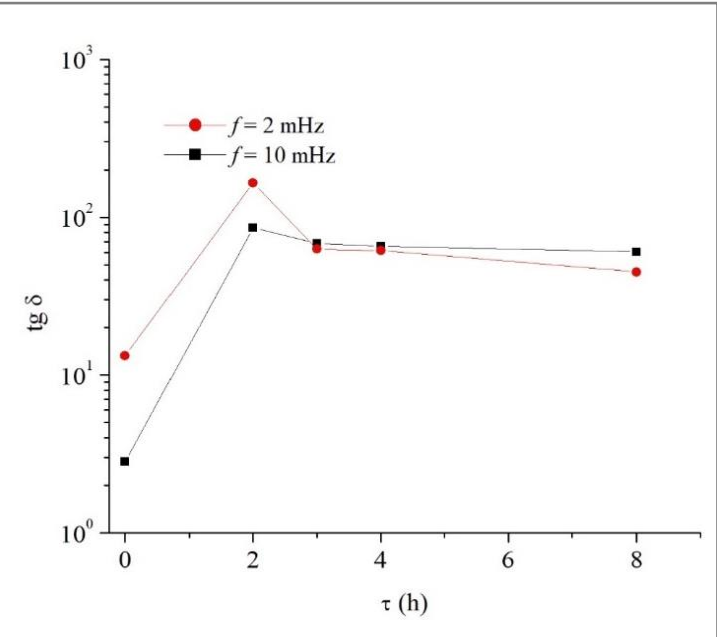

b)

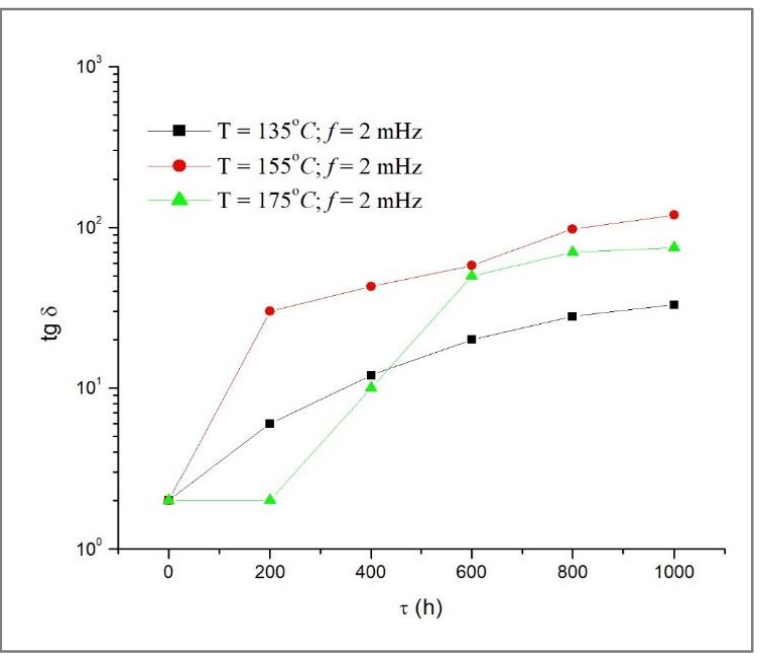

Fig. 5. Variation of dielectric loss factor as a function of aging time for a) Aging applied voltage $U=13 \mathrm{kV}$; b) Thermal aging temperatures $T=(135,155,175){ }^{\circ} \mathrm{C}$

Fig. $6 \mathrm{a}$ and Fig. $6 \mathrm{~b}$ show the variation of of the real part of complex relative permittivity, $\varepsilon \mathrm{r}$, as function of aging time, $\tau$, for $f=2 \mathrm{mHz}$, at a temperature $T=25^{\circ} \mathrm{C}$. Furthermore, when looking at these figures, for the oil accelerated aged under high electric field, a strong increase of $\varepsilon_{\mathrm{r}}{ }^{\prime}$ with the aging time, $\tau$, can be observed, for measurement frequency $f=2 \mathrm{mHz}$ and $f=10$ $\mathrm{mHz}$ respectively. This points to the existence of a very important degradation phenomenon, most likely explained by the processes that lead to the formation of polar free radicals that leads to higher polarization phenomena.
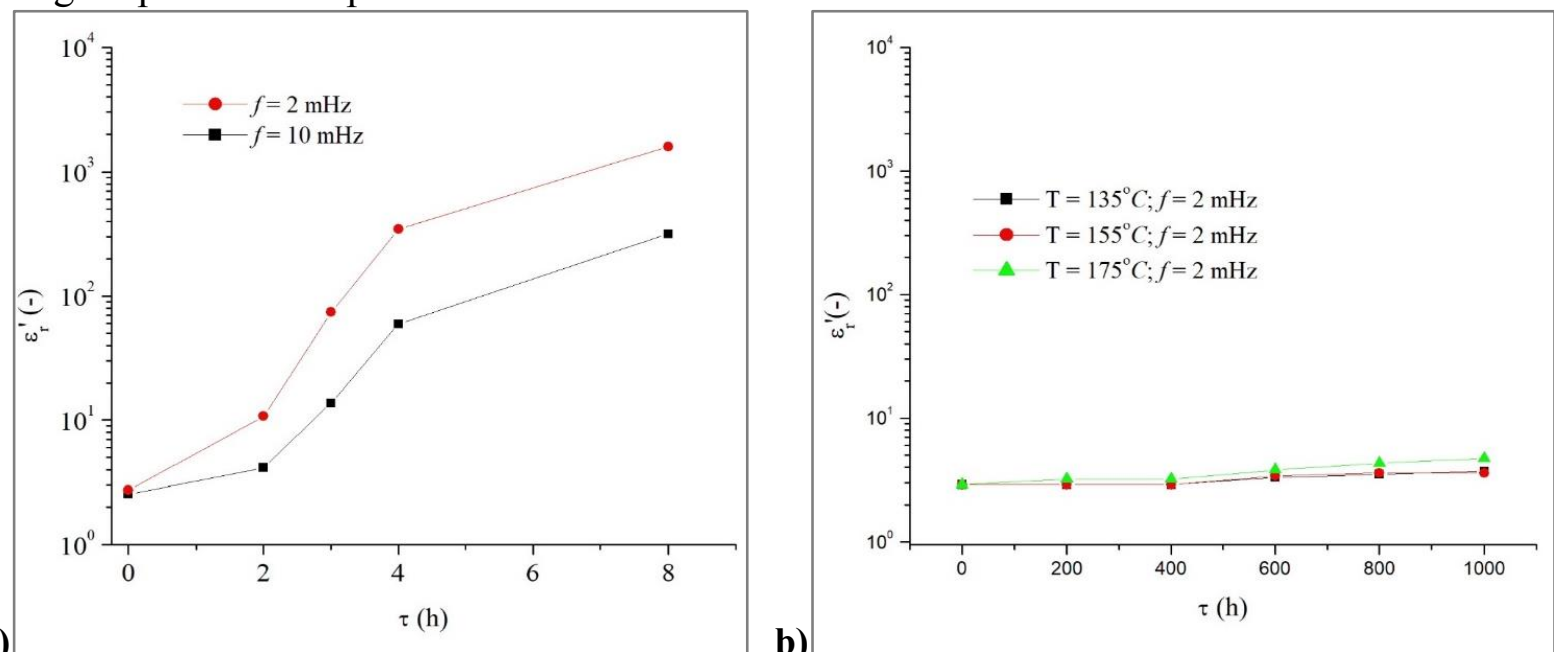

Fig. 6. Variation of the real part of complex relative permittivity as a function of aging time for a) Aging applied voltage $U=13 \mathrm{kV}$; b) Thermal aging temperatures $T=(135,155,175){ }^{\circ} \mathrm{C}$

Meanwhile, the variation of dielectric properties of mineral oil (i.e. the degradation process that corresponds to accelerated thermal aging), even for extremely long periods, is not so significant compared to the degradation under the action of high electric field, which proves that the action of the electric field and the partial discharge is very important and dramatically modifies the mineral oil condition.

\section{CONCLUSIONS}

The study presents two methods of accelerated aging of transformer mineral oil: one under the action of high electric field and partial discharges and the other by the exclusive action of the high temperature. Both methods are important in terms of assessing the condition of the 
insulation systems for power transformers. Focusing on the method of aging under electric field action allows to estimate, additionally, the impact of electric field and partial discharges on the oil condition.

Concerning the experimental method of accelerated ageing under high electric field and partial discharges it should be noted that the design of the aging cell considers several aspects among which the most important being the generation of gas bubbles in the oil under the action of electric field and the inception and preservation of an appropriate level of partial discharges in the gas bubbles.

Based on the experimental results presented in this study, it can be concluded that the thermal aging does not fully capture the processes and reactions that occur due of accelerated aging in the electric field.

It can be observed that in the case of mineral oil subjected to accelerated aging under high electric field and partial discharges, the real parts of the relative permittivity show very important increases comparing to the value obtained exclusively from thermal aging. This very large increase is most probably due to the fact that an important concentration of free polar radicals and free charge carriers are generated during the ageing process.

\section{REFERENCES}

[1] H. Zainuddin, "Transformer Oil Ageing Detection using Mach-Zender Interferometry Configuration as a Sensor", Int. J. of Recent Technology and Engineering, vol. 8, no. 26, pp. 826-831, 2019.

[2] A. Ciuriuc, M.S. Vihacencu, L.M. Dumitran and P.V. Noţingher, "Comparative Study on Power Transformer Vegetable and Mineral Oil Ageing”, Anals of the University of Craiova, no. 36, pp. 46-51, 2012.

[3] M. Martins and A. Gomes, "Comparative study of the thermal degradation of synthetic and natural esters and mineral oil: effect of oil type in the thermal degradation of insulating kraft paper", IEEE Electrical Insulation Magazine, vol. 28, no. 2, pp. 22-28, 2012.

[4] M. Yea, K. Han, J. Park, S. Lee and J. Choi, "Design optimization for the insulation of HVDC converter transformers under composite electric stresses", IEEE Transactions on Dielectrics and Electrical Insulation, vol. 25, no. 1, pp. 253-262, 2018. DOI: 10.1109/tdei.2018.006629.

[5] J. Kúdelčík, L. Varačka, E. Jahoda and S. Poljak, "Post-breakdown stages in transformer oil", The European Physical Journal Applied Physics, vol. 78, no. 2, pp. 208-213, 2017.

[6] J. Tanaka, "Free Radicals in Electrical Insulation", in Proceedings of the 19th Symposium of Electrical Insulating Materials", Osaka, Japan, 1986.

[7] I. Lingvay, V. Stănoi, L. Ungureanu, F. Șerban and A. Borș, "Gases forming during thermal ageing of some electrical use oils", Electrotehnica, Electronica, Automatica, vol. 65, no. 2, pp. 23-30, 2017.

[8] I. Fofana, J. Sabau, D. Bussieres and E. Robertson, "The Mechanism of Gassing in Power Transformer'", in Proceedings of the IEEE Int. Conf. on Dielectric Liquids (ICDL'80), 2008, pp. 1-4.

[9] I. Fofana, A. Bouaicha, M. Farzaneh, and J. Sabau, „Ageing Behavior of Mineral and Ester Liquids: A Comparative Study", 2008 Annual Report Conference on Electrical Insulation Dielectric Phenomena (CEIDP), Quebec, 26-29 October 2008, pp. 87-90.

[10] T.V. Oommen, "Vegetable Oils for Liquid-Filled Transformers", IEEE Electrical Insulation Magazine, Vol. 18, No. 1, January/February 2002, pp. 6-11.

[11]A. Ciuriuc, "Degradation and lifetime estimation of vegetable oil for power transformer", PhD Thesis, University Politehnica of Bucharest, 2015.

[12] W. Zaengl, "Dielectric spectroscopy in time and frequency domain for HV power equipment. I. Theoretical considerations", IEEE Electrical Insulation Magazine, vol. 19, no. 5, pp. 5-19, 2003. 